\title{
Effect of silicon and oxygen doping on donor bound excitons in bulk GaN
}

Galia Pozina, Sergey Khromov, Carl Hemmingsson, Lars Hultman and Bo Monemar

\section{Linköping University Post Print}

N.B.: When citing this work, cite the original article.

Original Publication:

Galia Pozina, Sergey Khromov, Carl Hemmingsson, Lars Hultman and Bo Monemar, Effect of silicon and oxygen doping on donor bound excitons in bulk GaN, 2011, Physical Review B. Condensed Matter and Materials Physics, (84), 16, 165213.

http://dx.doi.org/10.1103/PhysRevB.84.165213

Copyright: American Physical Society

http://www.aps.org/

Postprint available at: Linköping University Electronic Press

http://urn.kb.se/resolve?urn=urn:nbn:se:liu:diva-72660 


\title{
Effect of silicon and oxygen doping on donor bound excitons in bulk GaN
}

\author{
G. Pozina, ${ }^{*}$ S. Khromov, C. Hemmingsson, L. Hultman, and B. Monemar \\ Department of Physics, Chemistry and Biology, Linköping University, SE-581 83 Linköping, Sweden
}

(Received 27 July 2011; published 27 October 2011)

\begin{abstract}
Freestanding $n$-type intentionally doped GaN layers grown by halide vapor phase epitaxy (HVPE) were studied by transient photoluminescence (PL). Concentrations of silicon and oxygen were varied in the range between $10^{17}$ and $10^{18} \mathrm{~cm}^{-3}$, as confirmed by secondary ion mass spectroscopy (SIMS). We show that a reduction of the background silicon concentration by one order of magnitude compared to the background level in undoped samples can be achieved by incorporation of oxygen during the growth. A strong band gap narrowing (BGN) of $\sim 6 \mathrm{meV}$ was observed with increasing doping in the studied samples. The low temperature PL recombination time for donor-bound excitons (DBEs) was found to depend significantly on donor concentration. A model assuming generation of DBEs by capturing of free excitons by neutral donors explains the experimental results at low temperature. From fitting the experimental DBE lifetime to the model, the donor concentration dependence for $\mathrm{O}$ and $\mathrm{Si}$ donors could be reproduced. An effective exciton capture cross-section was found to be $\sim 9.4 \times$ $10^{-15}$ and $1.2 \times 10^{-14} \mathrm{~cm}^{2}$ for silicon and oxygen donors, respectively.
\end{abstract}

DOI: 10.1103/PhysRevB.84.165213

PACS number(s): 78.47.jd, 78.55.Cr

\section{INTRODUCTION}

Bulk GaN is significant as a substrate material for homoepitaxial growth of highly efficient GaN-based light-emitting diodes and laser diodes with long operation time. ${ }^{1}$ Using $\mathrm{GaN}$ substrates the threading dislocation density (TDD) in the nitride active layer in devices can be reduced to the order of TDD in the substrate. The development of GaN substrates with low defect and impurity densities is thus an important challenge for the semiconductor industry. Bulk GaN is also of fundamental interest since many phenomena can be studied regardless of stress or dislocation density in thick freestanding GaN layers. So far the most successful technique to produce large area GaN substrates with a relatively low concentration of impurities is halide vapor phase epitaxy (HVPE). ${ }^{2}$ High crystalline quality of HVPE GaN material allows detailed studies of optical phenomena related to properties of excitons and its electronic structure. ${ }^{3,4}$ A number of results has been reported about donor-bound exciton (DBE) recombination dynamics in GaN. ${ }^{5,6}$ At low temperatures, the photoluminescence (PL) decay of DBE transitions is determined mainly by the radiative recombination processes. However, previous studies have shown a large variation in the PL recombination time of DBEs, ${ }^{7,8}$ which partly can be explained by a poorer quality of stressed GaN layers grown heteroepitaxially. Despite a recent progress in the synthesis of the high-quality homoepitaxial or bulk GaN layers, the value of the radiative recombination time for $\mathrm{DBE}$ in $\mathrm{GaN}$ is still uncertain. The possible reason for that is the different level of residual (background) shallow impurities in the material. Unintentionally $n$-type doped HVPE GaN usually has donor concentrations of the order of $10^{17}-10^{18} \mathrm{~cm}^{-3}$. 9 Silicon and oxygen, the two main shallow donors in $\mathrm{GaN}$, are typically responsible for the rather high background impurity levels. Silicon is released into the HVPE reactor chamber during the growth due to etching of the quartz parts. Silicon has a slightly shallower energy level compared to oxygen and is more suitable for doping purposes, since it does not produce acceptor-like complexes with vacancies at the growth temperature and consequently impacts more efficiently the density of electrons in the active layer of devices.
However, a high concentration of oxygen, responsible for formation of undesirable deeper acceptor levels related to Gavacancy-oxygen $\left(\mathrm{V}_{\mathrm{Ga}}-\mathrm{O}_{\mathrm{N}}\right)$ complexes, ${ }^{10}$ is also detrimental. In addition a varying concentration of dopants in $\mathrm{GaN}$ substrates can cause instability of the growth process since the substrate temperature during epitaxial deposition depends on infrared absorption, which in turn depends on doping. Also, a high background concentration of impurities in substrates can result in an undesirably increased level of dopants in the active layer of devices. From this point of view it is critical for both device applications and for fundamental understanding to control concentration of donors in $\mathrm{GaN}$ substrates. In this paper we study how intentional doping by silicon and oxygen affects low temperature recombination of DBEs in HVPE bulk GaN. We demonstrate that the concentration of silicon in GaN can be reduced by using a moderate intentional doping of oxygen.

\section{EXPERIMENTAL DETAILS}

The fabrication of bulk GaN samples was done in a vertical $\mathrm{HVPE}$ reactor at $\sim 1000{ }^{\circ} \mathrm{C}-1040^{\circ} \mathrm{C}$ at atmospheric pressure on $2-\mu \mathrm{m}$-thick $\mathrm{GaN}$ templates grown by metal organic chemical vapor deposition (MOCVD) on 2-inch $c$-oriented sapphire substrates. For details about the reactor see Ref. 11. $\mathrm{N}_{2}$, or a mixture of $\mathrm{N}_{2}$ and $\mathrm{H}_{2}$, was used as a carrier gas. The $\mathrm{NH}_{3}$ and $\mathrm{HCl}$ flow rate was $21 / \mathrm{min}$ and $75 \mathrm{ml} / \mathrm{min}$, respectively, and the total carrier flow including precursor carrier gases was $17 \mathrm{l} / \mathrm{min}$. With these process parameters, the growth rate of $\mathrm{GaN}$ was about $150-200 \mu \mathrm{m} / \mathrm{h}$. GaN samples were intentionally doped. $200 \mathrm{ppm}$ of $\mathrm{O}_{2}$ diluted in $\mathrm{N}_{2}$ or 200 ppm of $\mathrm{SiH}_{4}$ diluted in $\mathrm{H}_{2}$ was mixed with a carrier gas to get a doping level in the range of $10^{17}-10^{18} \mathrm{~cm}^{-3}$. The average donor concentration for each sample was confirmed by secondary ion mass spectroscopy (SIMS) measurements and presented in Table I.

The dynamics of exciton recombination was studied by time-resolved PL (TRPL) with excitation by the third harmonics $\left(\lambda_{\mathrm{e}}=266 \mathrm{~nm}\right)$ from a Ti:sapphire femtosecond-pulsed laser with a frequency of $75 \mathrm{MHz}$. The laser power density was $100 \mathrm{~W} / \mathrm{cm}^{2}$. A Hamamatsu syncroscan streak camera 
TABLE I. Oxygen and silicon concentrations measured by SIMS in HVPE GaN samples.

\begin{tabular}{lcc}
\hline \hline Sample & Doping, $\mathrm{cm}^{-3}$ & \\
\hline & $\mathrm{O}$ & $\mathrm{Si}$ \\
B1 undoped & $1.0 \times 10^{16}$-background & $1.0 \times 10^{17}$-background \\
B2 & $1.7 \times 10^{16}$-background & $1.0 \times 10^{17}$ \\
B3 & $3.0 \times 10^{16}$-background & $1.3 \times 10^{17}$ \\
B4 & $2.2 \times 10^{16}$-background & $5.5 \times 10^{17}$ \\
B5 & $1.6 \times 10^{17}$ & $1.0 \times 10^{16}$-background \\
B6 & $4.1 \times 10^{17}$ & $1.4 \times 10^{16}$-background \\
B7 & $5.6 \times 10^{17}$ & $6.0 \times 10^{16}$-background \\
B8 & $8.2 \times 10^{17}$ & $2.0 \times 10^{16}$-background \\
\hline \hline
\end{tabular}

with a temporal resolution of $\sim 20$ ps was used for detection of the TRPL signal. Cross-sectional transmission electron microscopy (TEM) was made for the structural qualification of the bulk GaN material using an FEI Tecnai G2 $200 \mathrm{keV}$ FEG instrument.

\section{RESULTS}

The studied GaN layers are usually self-separated from the sapphire substrate and can be considered as stress-free quasi-bulk samples. In such thick layers we can neglect the influence of threading dislocations on the PL decay time. ${ }^{12}$ The density of structural defects decreases with increasing layer thickness, as shown in Fig. 1, where cross-sectional TEM images demonstrate a rapid evolution of crystal quality in $\mathrm{GaN}$ samples. We have found that after self-separation from the sapphire substrate, the interface region is severely defected and has very high dislocation and stacking fault density [Fig. 1(a)]. The TDD reduces from $\sim 10^{9} \mathrm{~cm}^{-2}$ in the 20 - $\mu$ m-thick layer [Fig. 1(b)] to $\sim 10^{6} \mathrm{~cm}^{-2}$ and to $\sim 5 \times 10^{5} \mathrm{~cm}^{-2}$ in the layer with the thickness of $400 \mu \mathrm{m}$ [Fig. 1(c)] and $1 \mathrm{~mm}$
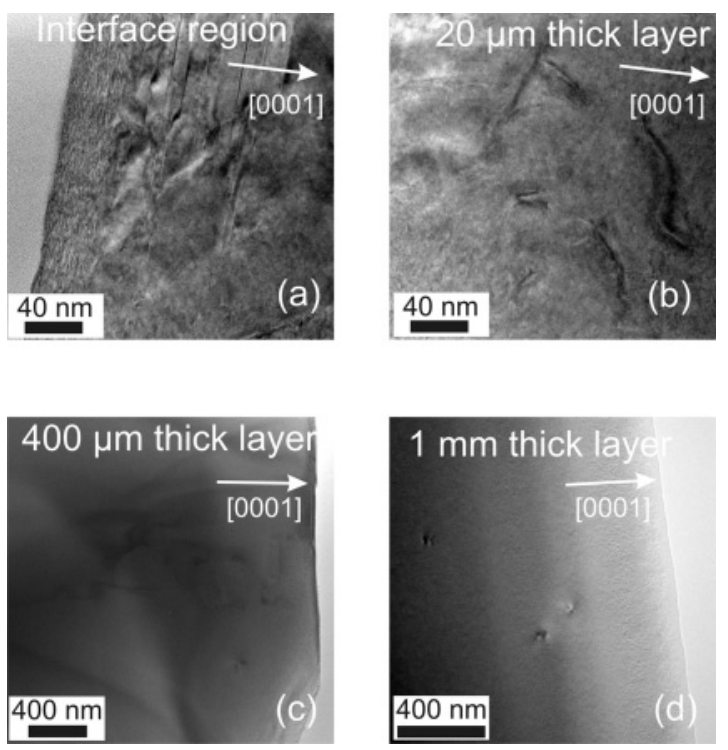

FIG. 1. Cross-sectional TEM images of the HVPE freestanding GaN samples showing (a) interface region after self-separation from the sapphire substrates, (b) typical area of a $20-\mu \mathrm{m}$-thick layer, (c) layer with thickness of $\sim 400 \mu \mathrm{m}$, and (d) a 1-mm-thick layer.

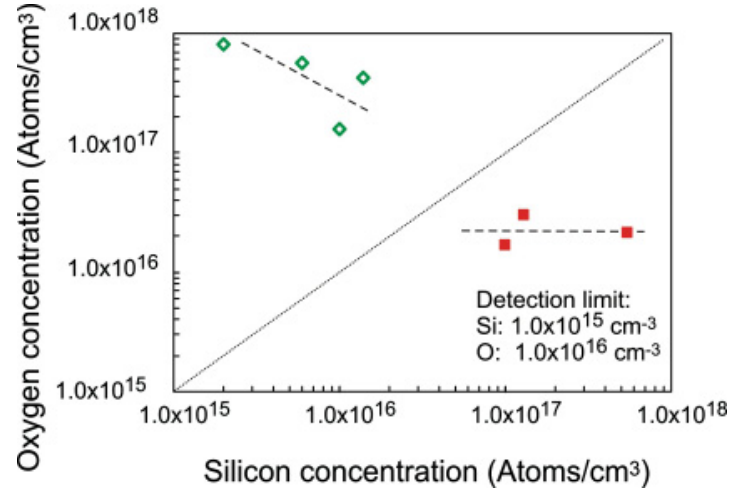

FIG. 2. (Color online) Oxygen concentration in GaN samples as a function of silicon concentration. Points correspond to the average values obtained by SIMS. Donor concentrations for the oxygen and silicon-doped GaN samples are shown by the open and closed signs, respectively. The dashed lines are guides to the eye.

[Fig. 1(d)], respectively. In the studied samples TDD was in the range of $\sim 10^{6} \mathrm{~cm}^{-2}$, which means that the distance between dislocations is much larger than the diffusion length of 60-80 nm related to excitons in GaN. ${ }^{13}$

In Fig. 2 an average oxygen concentration vs silicon concentration, determined by SIMS for each sample, is plotted. Open diamonds correspond to the GaN layers intentionally doped by oxygen, while closed squares are related to the samples doped by silicon. SIMS analysis revealed an interesting fact, namely, that intentional doping with oxygen reduces simultaneously the concentration of silicon donors, but not vice versa. By introducing oxygen in the range of $8 \times 10^{17} \mathrm{~cm}^{-3}$ we could decrease the concentration of silicon to $2 \times 10^{15} \mathrm{~cm}^{-3}$. This can be understood as follows. The concentration of $\mathrm{Ga}$ vacancies in $n$-type $\mathrm{GaN}$ is promoted by $\mathrm{O}$ doping, ${ }^{14}$ and this effect has been explained by formation of stable $\mathrm{V}_{\mathrm{Ga}}-\mathrm{O}_{\mathrm{N}}$ complexes during growth. In case of doping by both $\mathrm{Si}$ and $\mathrm{O}$, the $\mathrm{V}_{\mathrm{Ga}}$ will preferably bind to $\mathrm{O}$ since the binding energy of the $\mathrm{V}_{\mathrm{Ga}}-\mathrm{O}_{\mathrm{N}}$ complex in the range of $\sim 1.8 \mathrm{eV}$ is much larger than the binding energy of $0.23 \mathrm{eV}$ for the $\mathrm{V}_{\mathrm{Ga}}-\mathrm{Si}_{\mathrm{Ga}}$ complex. ${ }^{15}$ The $\mathrm{V}_{\mathrm{Ga}}-\mathrm{Si}_{\mathrm{Ga}}$ complex is therefore not stable at the growth temperature used. Thus, we suggest that the oxygen will suppress the incorporation of $\mathrm{Si}$ on $\mathrm{Ga}$ sites due to a competing process of $\mathrm{V}_{\mathrm{Ga}}-\mathrm{O}_{\mathrm{N}}$ formation. However, a high level of oxygen in $\mathrm{GaN}$ is undesirable since, as mentioned, it forms complexes with $\mathrm{Ga}$ vacancies responsible for carrier compensation and yellow luminescence (YL). Therefore, a relatively low doping by oxygen in the range of $\sim 1 \times 10^{17} \mathrm{~cm}^{-3}$ allows a rather drastic decrease of the Si concentration to $\sim 1 \times 10^{16} \mathrm{~cm}^{-3}$ without noticeably increasing YL.

The effect of doping on near-band gap emission properties was studied by TRPL. Time-integrated PL spectra measured at $2 \mathrm{~K}$ are shown in Fig. 3 for silicon- and oxygen-doped $\mathrm{GaN}$ layers. A typical PL spectrum for an undoped free-standing HVPE GaN sample is also presented for comparison in Fig. 3(a). Such bulk GaN samples are unintentionally $n$-type doped. PL spectra for samples with low concentration of silicon in Fig. 3(b) or oxygen in Fig. 3(c) are dominated by $\mathrm{DBE}$ recombination at $\sim 3.47 \mathrm{eV}$, which is very close to a typical DBE energy in freestanding unstrained $\mathrm{GaN}$ material. $^{5,9}$ The PL band in this case is an overlap of two 


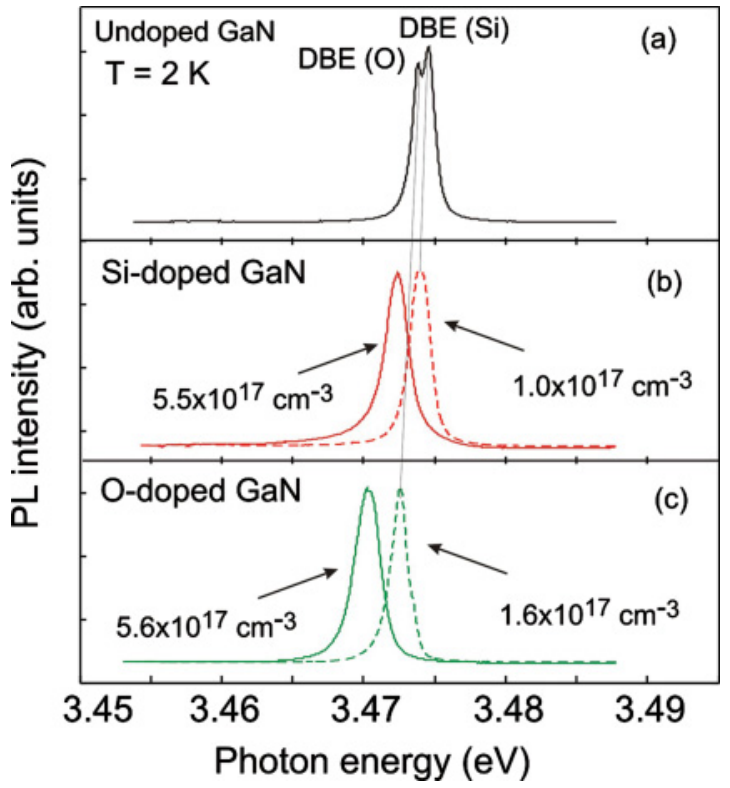

FIG. 3. (Color online) Low temperature (2 K) PL spectra for (a) undoped GaN layer, (b) two GaN layers intentionally doped by silicon, and (c) two GaN layers intentionally doped by oxygen. Donor concentrations are indicated in the plot.

peaks originated from transitions related to excitons bound to silicon and oxygen, respectively. These two peaks are clearly resolved in the undoped GaN sample, as shown in Fig. 3(a). A full width at half maximum (FWHM) of the conjugate DBE line in our samples is shown in Fig. 4 as a function of the total shallow donors concentration, i.e., as the sum of oxygen and silicon concentrations determined by SIMS. The FWHM depends on doping concentration and varies from $1.3 \mathrm{meV}$ to $2.3 \mathrm{meV}$ for the lowest and highest doped $\mathrm{GaN}$ layer, respectively. The line broadening is expected due to an increasing donor density and consequently with the enhanced overlap of their wave functions. This is also consistent with a pronounced asymmetric Lorentzian shape of the DBE line for samples with the donor concentrations of $5 \times 10^{17} \mathrm{~cm}^{-3}$ or higher. It can indicate that the homogeneous broadening of the bound exciton becomes comparable to the inhomogeneous broadening caused by strain and structural imperfections

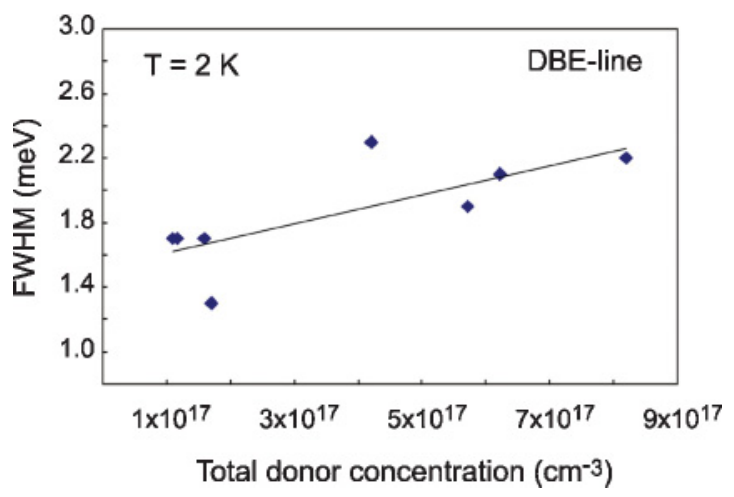

FIG. 4. (Color online) PL line width at low temperature as the function of the total donor concentration. The solid line is the guide to the eye. when the PL line shape is determined by overlapping several Lorentzian curves and the cumulative curve can be described by the Gaussian function. Though the Lorentzian lineshape is related to the radiative lifetime of the DBE states at $2 \mathrm{~K}$, several nonradiative decay processes such as multiphonon emission or tunneling of the excitons or holes to another impurity center might contribute to the experimental lifetime. However, it is shown by Sturge et al. (Ref. 16) for N-doped GaP that the quantum efficiency at $2 \mathrm{~K}$ can be considered as close to $100 \%$ with good accuracy.

We have observed a relatively large shift of the DBE line position to the lower energy with increasing donor concentrations, which is in qualitative agreement with results for cubic GaN. ${ }^{17}$ The maximal change of the DBE peak energy was found to be as large as $6 \mathrm{meV}$ at the maximal doping level of $8.8 \times 10^{17} \mathrm{~cm}^{-3}$ in our samples. This result can be explained by the band gap narrowing (BGN). We have estimated the BGN using the model developed by Jain et al. ${ }^{18}$ for $n$ - and $p$-type semiconductors. For $n$-type GaN we write the following equation for the BGN:

$$
\frac{\Delta E_{g}}{R^{*}}=\frac{1.83}{r_{s}}+\frac{0.95}{r_{s}^{3 / 4}}+\frac{\pi}{2} \frac{1}{r_{s}^{3 / 4}}\left(1+\frac{m_{h}^{*}}{m_{e}^{*}}\right) .
$$

Here, $R^{*}$ is the effective Rydberg energy

$$
R^{*}=m_{e}^{*} e^{4} / 2 \hbar^{2} \varepsilon^{2},
$$

and $r_{s}$ is the average distance between electrons, normalized to effective Bohr radius

$$
r_{s}=\frac{\left(\frac{3}{4} \pi N\right)^{1 / 3} m_{e}^{*} e^{2}}{4 \pi \varepsilon \hbar^{2}}
$$

where $m_{e}^{*}$ and $m_{h}^{*}$ are electron and hole effective masses, respectively. The first term in Eq. (1) was obtained from the exchange energy of the majority carriers, the second and the third terms are correlation and impurity interaction energy, respectively. ${ }^{18}$ We have calculated the BGN according to Eq. (1) using $0.18 m_{0}$ and $1.61 m_{0}$ for the electron and heavy hole effective masses, respectively. ${ }^{19}$ The DBE peak position is expressed as

$$
E_{\mathrm{DBE}}=E_{\mathrm{DBE}}^{0}-\Delta E_{\mathrm{g}},
$$

where $E_{\mathrm{DBE}}^{0}$ is related to the peak position energy of the DBE in undoped GaN. Results of calculations together with experimental PL peak energies are shown as a function of donor concentrations for samples doped with silicon and oxygen in Figs. 5(a) and 5(b), respectively. The model is in a good qualitative agreement with experimental data and supports the large effect of BGN in GaN even at moderate doping concentrations. The shift of the PL peak to lower energies becomes even more pronounced in highly doped $\mathrm{GaN}$ layers ${ }^{20}$ however, for carrier concentrations above the Mott density, the Burstein-Moss shift of the absorption edge to the higher energies has to be taken into account. ${ }^{21,22}$ Since we study here relaxed bulk HVPE GaN we can likely neglect the strain effect on the PL peak position observed in both undoped and Si-doped samples. ${ }^{23,24}$

Taking into account the large influence of doping on the position of the DBE PL line, we have examined the 

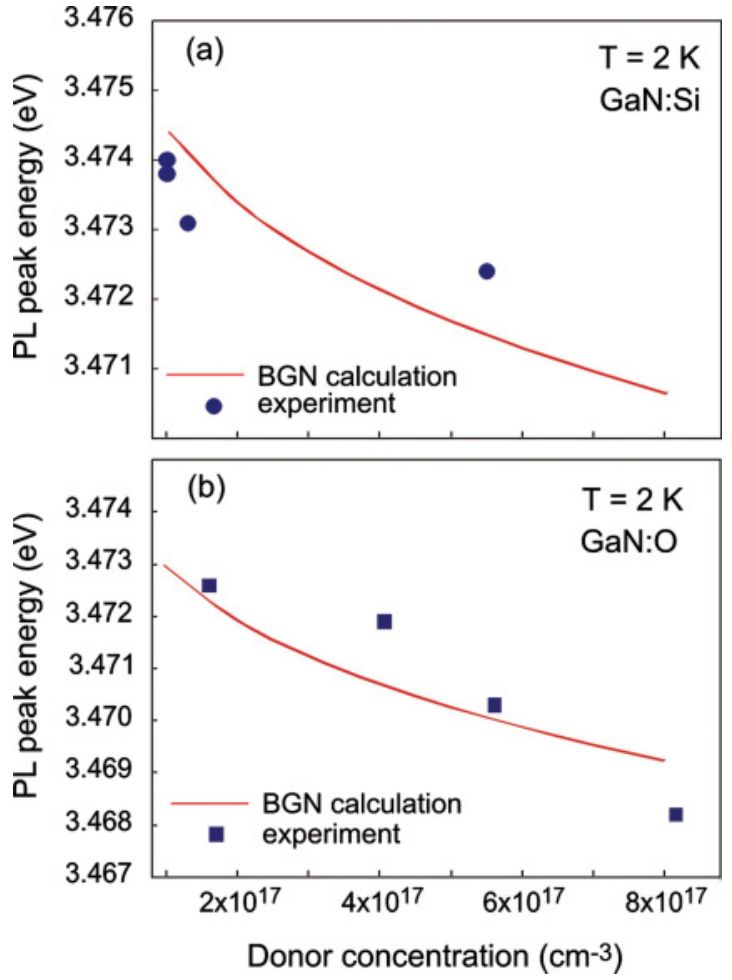

FIG. 5. (Color online) DBE PL peak energy vs (a) silicon and (b) oxygen concentrations. The experimental points are shown by signs and the calculations according Eq. (2) by the solid lines.

effect of doping on the PL lifetime of the DBE. Analysis of low temperature TRPL revealed a rather different transient behavior of PL for the DBE transitions depending on doping in the samples. The recombination time $\tau$ can be expressed as contribution of radiative $\left(\tau_{\mathrm{r}}\right)$ and nonradiative $\left(\tau_{\mathrm{nr}}\right)$ times as follows:

$$
1 / \tau=1 / \tau_{\mathrm{r}}+1 / \tau_{\mathrm{nr}}
$$

Nonradiative recombination channels become significant at elevated temperatures since they are typically activated according to $\tau_{\mathrm{nr}}=A \exp \left(-E_{\mathrm{a}} / \mathrm{kT}\right)$. Indeed, studies of the DBE thermal behavior have shown that the PL recombination time decreases rapidly for temperatures above $20 \mathrm{~K}$, as reported previously in GaN. ${ }^{9,25}$ Thus, at the low temperature of $2 \mathrm{~K}$ the DBE PL lifetime is determined mainly by the radiative recombination time. A number of low-temperature PL decay curves measured at the DBE peak position are plotted for the GaN layers doped with Si and oxygen in Figs. 6(a) and 6(b), respectively. We obtain the DBE lifetime from fitting of the PL transient data using a single exponential decay law, as shown by thick solid lines in Fig. 6. Extracted values (shown by signs) are plotted as a function of doping concentration in Fig. 7. It is clear that the PL decay times at $2 \mathrm{~K}$ for the DBE transition depends on the identity of the dopant atom (i.e., if it is silicon or oxygen) and on the donor concentration. Undoubtedly, the recombination time decreases with increasing silicon and/or oxygen concentrations in the HVPE GaN layers. Subsequently we consider a model describing the experimental results.

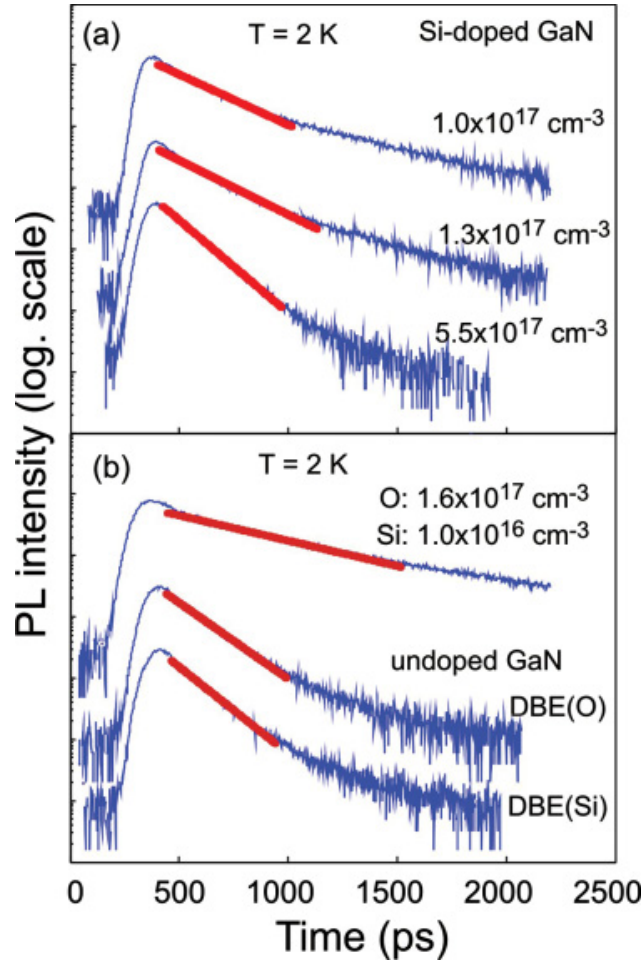

FIG. 6. (Color online) (a) Low-temperature PL decay curves measured at the DBE PL peak energy for the GaN layers doped by silicon. The graphs are vertically shifted for clarity. (b) The PL decay curves at $2 \mathrm{~K}$ shown for the DBEs in the undoped and in the oxygen-doped $\mathrm{GaN}$ samples. Thick solid lines show fitting by a single exponential decay law.

\section{MODELING OF THE DBE PL TRANSIENT RESULTS}

In $\mathrm{GaN}$, which is a direct wide bandgap semiconductor, the radiative recombination rate for free excitons (FE) is high. However, the main radiative recombination processes at low temperatures, as it is seen from the PL spectra, occur for the bound excitons (in our case, DBEs). An accurate quantitative analysis is complicated since the time dependencies of the

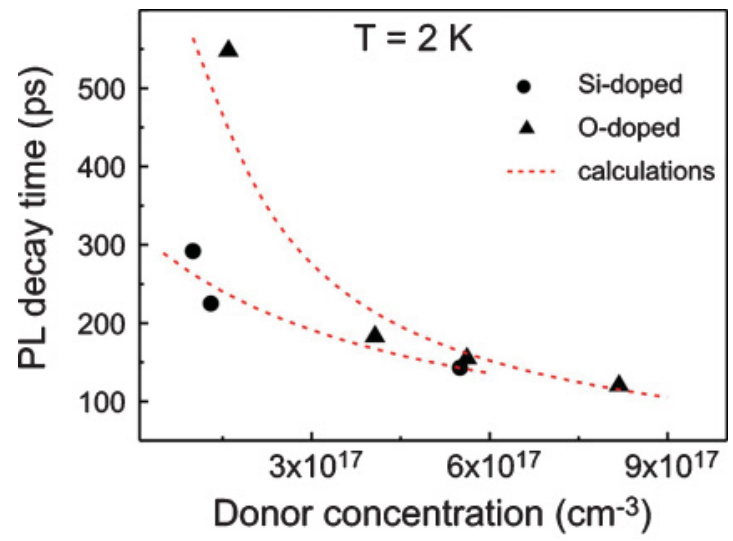

FIG. 7. (Color online) DBE lifetime at $2 \mathrm{~K}$ extracted from the fit of the experimental PL decay curves vs donor concentration. The data obtained for the silicon- and oxygen-doped GaN samples are shown by circles and triangles, respectively. Theoretical fitting according to Eq. (12) is shown by the dashed lines. 
concentrations for carriers, excitons, and neutral donors have to be described using a set of nonlinear differential equations presented, for example, in Ref. 26. However, the analytical expression for the radiative lifetime in Ref. 26 was obtained for a very simplified model considering a coupled system described by one common radiative decay rate. We instead use the following approach. The PL rise time $\left(\tau_{r}\right)$ for the bound excitons is longer than the PL rise time for free excitons. In addition with the absence of the FE line in PL in our samples, it allows us to assume that after excitation a number of free excitons can be formed, which then can be quickly trapped by donors. Thus, the main process of the DBE formation is caused by capturing of FEs. For simplicity, we consider only the donors with a dominating concentration. Then, for the temporal change of the bound exciton concentration $n_{b}$ we can write

$$
\frac{d n_{b}}{d t}=-\gamma n_{b}+r_{i}-g_{i T} .
$$

Here, $\gamma$ is a radiative recombination rate of bound excitons, $g_{i T}$ is the re-emission of FE by a donor, and $r_{i}$ is the capture rate of free excitons by donors and depends both on concentrations of FE, $n$, and the unoccupied neutral donors, which still can capture an FE. Thus, we can express

$$
r_{i}=\alpha N_{i}[1-f] n .
$$

Here, $N_{i}$ is the total concentration of neutral donors, $N_{i}[1-f]$ is the concentration of neutral donors without captured excitons, $f$ is the probability to capture an FE, and $\alpha$ is the capture coefficient for donors.

For re-emission we use

$$
g_{i T}=\beta N_{i} f .
$$

Here, $\beta$ is the reemission coefficient, which depends on temperature and the binding energy of the exciton to donor, and $N_{i} f$ is the concentration of donors with captured excitons, i.e., DBE, $n_{b}$.

We may assume that equilibrium is established between free and bound excitons even at nonselective excitation, at least if the temperature is not too low. Then, from the detailed balance between capture and emission processes we have the relation between $\alpha$ and $\beta$,

$$
r_{i}=g_{i T},
$$

and consequently, using Eqs. (4) and (5), we write

$$
\alpha N_{i}\left[1-f_{0}\right] n_{0}=\beta N_{i} f_{0} .
$$

Here, $f_{0}$ is an equilibrium probability to capture free excitons, which behave generally as bosons and consequently follow Bose-Einstein statistics $f_{0}=\frac{1}{e^{(E-\mu) / k T}-1}$, where $\mu$ is the chemical potential for an exciton. ${ }^{27}$ Since the exciton density is rather low in our samples we can approximate with the Maxwell-Boltzmann law $f_{0}=\frac{1}{e^{(E-\mu) / k T}}$ to describe $f_{0}$ even at low temperatures. Consequently, $f_{0}$ is expressed as

$$
f_{0}=\frac{n_{0}}{n_{1}}
$$

where the term $n_{1}=n_{0} e^{-(E-\mu) / k T}$ depends on ionization energy of donors and temperature.
Then, Eq. (6) gives

$$
\beta=\alpha \frac{1-f_{0}}{f_{0}} n_{0}=\alpha\left(n_{1}-n_{0}\right) .
$$

We assume in our model that FE and DBE have the same spatial distribution, and that the emission comes from the region of the excitation $(\sim 50 \mathrm{~nm}$ under the experimental conditions), which is reasonable in the case of the doped samples in distinction from undoped $\mathrm{GaN}{ }^{8}$

Thus, Eq. (3) can be written as

$$
N_{i} \frac{d f}{d t}=-\gamma N_{i} f+\alpha N_{i} n(1-f)-\alpha N_{i}\left(n_{1}-n_{0}\right) f .
$$

In Eq. (9) we express $n=n_{0}+\delta n$ and $f=f_{0}+\delta f$, where $\delta n$ and $\delta f$ are the deviation of $\mathrm{FE}$ and probability to capture excitons, respectively, from equilibrium conditions.

Taking into account Eq. (7) we get

$$
\begin{aligned}
N_{i} \frac{d \delta f}{d t}= & -\gamma N_{i} f_{0}-\gamma N_{i} \delta f+\alpha N_{i} \delta n\left(1-f_{0}\right) \\
& -\alpha N_{i}\left(n_{1}+\delta n\right) \delta f .
\end{aligned}
$$

At low temperature we can assume that $\delta n+N_{i} \delta f=0$, which means that free excitons are captured by donors before they recombine radiatively, which is reasonable for our doped samples that show no FE-related line in the PL spectra. Thus, we write Eq. (9) as

$$
\begin{aligned}
N_{i} \frac{d \delta f}{d t}= & -\gamma N_{i} f_{0}-N_{i} \delta f\left(\gamma+\alpha N_{i}\left(\left(1-f_{0}\right)\right.\right. \\
& \left.\left.+n_{1}-N_{i} \delta f\right)\right) .
\end{aligned}
$$

The term $\gamma+\alpha N_{i}\left(\left(1-f_{0}\right)+n_{1}-N_{i} \delta f\right)$ describes the recombination rate of DBEs. Assuming that $\delta f$ is small compared to the equilibrium value, we get a recombination time for the DBE, which can be expressed in the following form:

$$
\tau=\frac{1}{\gamma+\alpha\left(N_{i}^{0}+n_{1}\right)} .
$$

The term $N_{i}^{0}=N_{i}\left(1-f_{0}\right)$ describes equilibrium concentration of neutral donors, which can capture free excitons. The model takes into account only one type of donor, which is a reasonable approximation in our case, when samples were intentionally doped with concentrations exceeding background by one order of magnitude. Theoretical predictions of the radiative lifetime for bound excitons within the spherical effective-mass approximation for different III-V semiconductors such as GaAs, InAs, and InP were done by $\mathrm{G}$. D. Sanders et al. (Ref. 28). Comparison to the experimental data for the DBE has revealed a disagreement within an order of magnitude. The discrepancy was explained by the larger number of the DBE rotational excited states. ${ }^{29}$

The best fitting of experimental recombination time using Eq. (12) is shown in Fig. 7 by the dashed lines. The obtained fitting parameters $\gamma+\alpha n_{1}$ and $\alpha\left(1-f_{0}\right)$ can be used to estimate the low limit for the radiative recombination time $\left(\tau_{r}=\frac{1}{\gamma}\right)$ for DBE and the upper limit for the exciton capture cross-section $S\left(\alpha=S v_{T}\right)$ at $2 \mathrm{~K}$, respectively. This rough estimation suggests that the radiative time for silicon and oxygen donor is exceeding 320 ps and $1.25 \mathrm{~ns}$, respectively. This is consistent with the results for lower doping concentrations in Ref. 8. The 
upper limits of the effective exciton capture cross-section for the silicon and oxygen donor were determined to $9.4 \times 10^{-15}$ and $1.2 \times 10^{-14} \mathrm{~cm}^{2}$, respectively, using a free exciton thermal velocity of $7.1 \mathrm{~nm} / \mathrm{ps}$ at $2 \mathrm{~K}$ obtained from

$$
v_{T}=\sqrt{3 k_{B} T / m_{e x}} .
$$

Here, an exciton effective mass $m_{e x}$ in wurtzite GaN was calculated using the electron and the heavy hole effective masses of $0.18 m_{0}$ and $1.61 m_{0}$, respectively. ${ }^{19}$

\section{CONCLUSIONS}

We have studied intentionally doped $n$-type GaN freestanding layers grown by HVPE. Silicon and oxygen were used as dopant atoms. We find that a moderate doping in the range of $10^{17} \mathrm{~cm}^{-3}$ results in a drastic decrease of the silicon doping to $10^{16} \mathrm{~cm}^{-3}$, which is less than a typical background Si-doping concentration of $10^{17} \mathrm{~cm}^{-3}$ in the undoped $\mathrm{GaN}$ samples. It appears that oxygen suppresses the incorporation of $\mathrm{Si}$ on $\mathrm{Ga}$ sites by a competing process of Ga-vacancy-O complex formation during growth. There is a strong effect of doping on BGN revealed by a $6 \mathrm{meV}$ lower energy shift of the DBE peak position while doping increases only one order of magnitude. A significant influence of doping was demonstrated also on the low temperature PL lifetime of the DBE. We have suggested a model explaining the dependence of the low temperature recombination rate for the DBE transition on doping concentration. From fitting the experimental lifetime for the DBE, a lower limit for the radiative time of exciton bound to $\mathrm{Si}$ and $\mathrm{O}$ is estimated to $320 \mathrm{ps}$ and $1.25 \mathrm{~ns}$, respectively. The upper limits of the effective capture cross-section are $9.4 \times 10^{-15}$ and $1.2 \times$ $10^{-14} \mathrm{~cm}^{2}$ for $\mathrm{Si}$ and $\mathrm{O}$ donors, respectively.

\section{ACKNOWLEDGMENTS}

This work was supported by the Swedish Research Council (VR), the Swedish Energy Agency, and The Swedish Governmental Agency for Innovation Systems (VINNOVA).
*Corresponding author: galia@ifm.liu.se

${ }^{1}$ P. Gibart, Rep. Prog. Phys. 67, 667 (2004).

${ }^{2}$ C. Hemmingsson, P. P. Paskov, G. Pozina, M. Heuken, B. Schineller,

B. Monemar, J. Cryst. Growth 300, 32 (2007).

${ }^{3}$ T. V. Shubina, M. M. Glazov, A. A. Toropov, N. A. Gippius, A. Vasson, J. Leymarie, A. Kavokin, A. Usui, J. P. Bergman, G. Pozina, and B. Monemar, Phys. Rev. Lett. 100, 087402 (2008).

${ }^{4}$ B. Monemar, P. P. Paskov, J. P. Bergman, A. A. Toropov, T. V. Shubina, T. Malinauskas, and A. Usui, Phys. Status Solidi B 245, 1723 (2008).

${ }^{5}$ A. Wysmolek, K. P. Korona, R. Stepniewski, J. M. Baranowski, J. Błoniarz, M. Potemski, R. L. Jones, D. C. Look, J. Kuhl, S. S. Park, and S. K. Lee, Phys. Rev. B 66, 245317 (2002).

${ }^{6}$ K. P. Korona, A. Wysmolek, R. Stepniewski, J. Kuhl, D. C. Look, S. K. Lee, and J. Y. Han, J. Lumin. 112, 30 (2005).

${ }^{7}$ B. Monemar, P. P. Paskov, J. P. Bergman, A. A. Toropov, T. V. Shubina, and A. Usui, Superlattices Microstruct. 43, 610 (2008).

${ }^{8}$ B. Monemar, P. P. Paskov, J. P. Bergman, G. Pozina, A. A. Toropov, T. V. Shubina, T. Malinauskas, and A. Usui, Phys. Rev. B 82, 235202 (2010).

${ }^{9}$ G. Pozina, C. Hemmingsson, J. P. Bergman, D. Trinh, L. Hultman, and B. Monemar, Appl. Phys. Lett. 90, 221904 (2007).

${ }^{10}$ D. C. Reynolds, D. C. Look, B. Jogai, J. E. Van Nostrand, R. Jones, and J. Jenny, Solid State Commun. 106, 701 (1998).

${ }^{11}$ C. Hemmingsson, P. P. Paskov, G. Pozina, M. Heuken, B. Schineller, and B. Monemar, Superlattices Microstruct. 40, 205 (2006).

${ }^{12}$ G. Pozina, C. G. Hemmingsson, J. P. Bergman, D. Trinh, L. Hultman, and B. Monemar, Superlattices Microstruct. 43, 605 (2008).

${ }^{13}$ N. Pauc, M. R. Phillips, V. Aimez, and D. Drouin, Superlattices Microstruct. 40, 557 (2006).
${ }^{14}$ J. Oila, V. Ranki, J. Kivioja, K. Saarinen, P. Hautojärvi, J. Likonen, J. M. Baranowski, K. Pakula, T. Suski, M. Leszczynski, and I. Grzegory, Phys. Rev. B 63, 045205 (2001)

${ }^{15}$ J. Neugebauer and C. G. Van de Walle, Appl. Phys. Lett. 69, 503 (1996).

${ }^{16}$ M. D. Sturge, E. Cohen, and K. F. Rodgers, Phys. Rev. B 15, 3169 (1977).

${ }^{17}$ Z. Q. Li, H. Chen, H. F. Liu, L. Wan, M. H. Zhang, Q. Huang, J. M. Zhou, N. Yang, K. Tao, Y. J. Han, and Y. Luo, Appl. Phys. Lett. 76, 3765 (2000).

${ }^{18}$ S. C. Jain, J. M. McGregor, and D. J. Roulston, J. Appl. Phys. 68, 3747 (1990).

${ }^{19}$ M. Suzuki and T. Uenoyama, Solid-State Electron. 41, 271 (1997).

${ }^{20}$ M. Yoshikawa, M. Kunzer, J. Wagner, H. Obloh, P. Schlotter, R. Schmidt, N. Herres, and U. Kaufmann, J. Appl. Phys. 86, 4400 (1999).

${ }^{21}$ S. Shokhovets, K. Köhler, O. Ambacher, and G. Gobsch, Phys. Rev. B 79, 045201 (2009).

${ }^{22}$ H. P. D. Schenk, S. I. Borenstain, A. Berezin, A. Schön, E. Cheifetz, S. Khatsevich, and D. H. Rich, J. Appl. Phys. 103, 103502 (2008).

${ }^{23}$ G. Pozina, N. V. Edwards, J. P. Bergman, T. Paskova, B. Monemar, M. D. Bremser, and R. F. Davis, Appl. Phys. Lett. 78, 1062 (2001).

${ }^{24}$ A. Cremades, L. Görgens, O. Ambacher, M. Stutzmann, and F. Scholz, Phys. Rev. B 61, 2812 (2000).

${ }^{25}$ G. Pozina, J. P. Bergman, T. Paskova, and B. Monemar, Appl. Phys. Lett. 75, 4124 (1999).

${ }^{26}$ O. Brandt, J. Ringling, K. H. Ploog, H.-J. Wünsche, and F. Henneberger, Phys. Rev. B 58, R15977 (1998).

${ }^{27}$ J. Bisquert, Chem. Phys. Lett. 462, 229 (2008).

${ }^{28}$ D. Sanders and Y. C. Chang, Phys. Rev. B 28, 5887 (1983).

${ }^{29}$ E. Finkman, M. D. Sturge, and R. Bhat, J. Lumin. 35, 235 (1986). 